
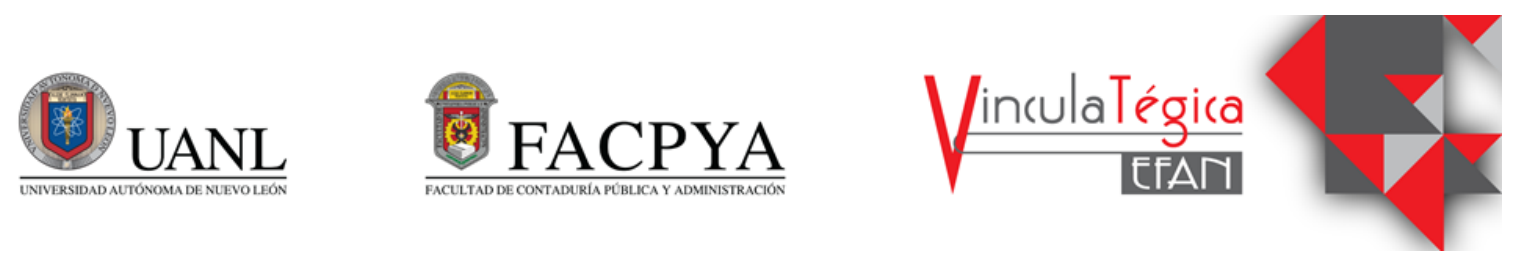

\title{
Elementos esenciales que garantizan el gobierno abierto: diagnóstico mexicano
}

\author{
Karina Galván Zavala ${ }^{1}$, Artemio Jiménez Rico $^{2}$ y Perla Cristina Laguna Córdoba ${ }^{3}$ \\ ${ }^{1}$ Universidad de Guanajuato, División de Ciencias Económico Administrativas, k.galvan@ugto.mx, \\ Fraccionamiento 1; Col. El Establo S/N; C.P. 36250 Guanajuato, México, 014737352900 ext. 2858. \\ ${ }^{2}$ Universidad de Guanajuato, División de Ciencias Económico Administrativas, a.jimenezrico@ugto.mx, \\ Fraccionamiento 1, Col. El Establo S/N, C.P. 36250 Guanajuato, México, 014737352900 \\ ${ }^{3}$ Universidad de Guanajuato, División de Ciencias Económico Administrativas, p.laguna@ugto.mx, \\ Fraccionamiento 1, Col. El Establo S/N; C.P. 36250 Guanajuato, México, 014737352900
}

Información del artículo revisado por pares

Fecha de aceptación: junio-2021

Fecha de publicación en línea: diciembre-2021

DOI: https://doi.org/10.29105/vtga7.1-99

\section{Resumen}

Ante la creciente problemática de corrupción e impunidad albergada en instituciones públicas y privadas a nivel global, surgen mecanismos que propician la transparencia, rendición de cuentas para dar paso al gobierno abierto, tal es el surgimiento en 2015 de la Ley General de Transparencia y acceso a la información para el Estado mexicano, cuya reforma más reciente se da en el año 2020, lo que resulta positivo en el establecimiento de los principios, las bases generales, los procedimientos en garantizar el derecho de acceso a la información en posesión de organismos públicos y cualquier figura que reciba y ejerza recursos públicos.

Sin embargo, para considerar que dichos mecanismos tienen efectos positivos en el fomento de gobierno abierto se requiere la participación de la ciudadanía, actualmente nos encontramos con escasa participación ciudadana, desconocimiento del derecho, usos y fines de la información pública. el presente documento estudia los elementos esenciales que garantizan el gobierno abierto, los modelos de gobierno abierto y recoge un diagnóstico de gobierno abierto en México con la finalidad de detectar cuales de los elementos de gobierno abierto presentan desarrollo y propiciar recomendaciones que aporten al incremento de la participación de la ciudadanía en la vida pública. la métrica de gobierno abierto 2019 para México en 2019 revela que el promedio nacional de gobierno abierto en una escala de 0 a 1 es 0.52
Abstract

Faced with the growing problem of corruption and impunity harbored in public and private institutions at a global level, mechanisms arise that promote transparency, accountability to give way to open government, such is the General Law of Transparency and access to the information for the Mexican State, which is positive in the establishment of the principles, the general bases, the procedures in guaranteeing the right of access to information in possession of public institutions and any figure that uses and exercises public resources.

However, to consider that these mechanisms have positive effects on the promotion of open government requires citizen participation, currently we find little citizen participation, ignorance of the law, uses and fines of public information. This document studies the essential elements by open government, open government models and includes a diagnosis of open government in Mexico in order to detect which of the open government elements present development and provide recommendations that contribute to the increase of the citizen participation in public life. The national overage of open government 2019 index in México, on a scale of 0 to 1 , is 0.52 
Palabras clave: Transparencia, gobierno abierto, participación ciudadana

\section{INTRODUCCIÓN}

Para discutir los la relación entre trasparencia gubernamental, participación ciudadana, rendición de cuentas y la incorporación de tecnología e innovación en el proceso del gobierno abierto, es importante establecer una definición de gobierno abierto, por lo que se presentan las principales definiciones que contienen la naturaleza y propósito del gobierno abierto.

Para Carlos Guardan (2010) el gobierno abierto como la doctrina política que sostiene que la actividad del gobierno y de la administración del estado debe estar abierta a todos los niveles de escrutinio eficaz del público y de su supervisión. (Chaidez Castaños , 2016)

Cesar Calderón (2010) un gobierno abierto trata de constante conversación con los ciudadanos, en donde se escucha de manera directa aquello que demandan para integrarlos a la toma de decisiones y también, "facilita la colaboración de los ciudadanos y funcionarios en el desarrollo de los servicios que presta y que comunica que todo lo que decide y hace de forma abierta y transparente. (Chaidez Castaños , 2016)

Para Valenzuela y García-Gil Gobierno abierto se refiere a una forma muy particular de gobernar y gestionar las políticas públicas, caracterizada por la transparencia, la participación ciudadana y la colaboración entre gobiernos y ciudadano. El cual coexiste con otros actores de la sociedad civil y el mercado, otorgando validez a nuevos mecanismos y estrategias para decidir los asuntos públicos de forma conjunta.

Para Sandoval Almanza, gobierno abierto debe entenderse como una plataforma tecnológica institucional que convierta los datos gubernamentales en datos abiertos para permitir su uso, protección y colaboración por parte de los ciudadanos en los procesos de decisión pública, rendición de cuentas y mejoramiento de los servicios públicos. (Sandoval Almazan, 2015)

Para el instituto nacional de
Keywords: Transparency, open government, citizen participation JEL:G38

transparencia, acceso a la información y protección de datos personales, el gobierno abierto es un esquema de gestión y producción de políticas orientado a la atención y solución colaborativa de los problemas públicos con base en colegiados plurales y, en cuyo trabajo convergen la transparencia y la participación ciudadana como criterios básicos, buscando propiciar un ambiente de rendición de cuentas e innovación social.

\section{PRINCIPIOS DE GOBIERNO}

\section{ABIERTO}

De acuerdo con la alianza para el gobierno abierto, los principios fundamentales del Gobierno Abierto son los siguientes:

Transparencia: La información sobre las decisiones y el quehacer gubernamental deben ser abiertas, completas, oportunas, gratuitas y de fácil acceso para el público. Ello supone que los datos públicos deben cumplir con parámetros y estándares comúnmente reconocidos, tales como estar disponibles en formato brutos, susceptibles de ser procesados, que puedan ser accesibles a través de herramientas tecnológicas y de comunicación, etc.

Participación Ciudadana: Los gobiernos deberán buscar que la ciudadanía se interese e involucre en el debate público, proveyendo los canales apropiados (aportando información y espacios de consulta) y mediante contribuciones que conduzcan a una gobernanza más efectiva, innovadora, responsable y que atienda las necesidades de la sociedad.

Rendición de cuentas: Supone la existencia de normativas, procedimientos y mecanismos para que los servidores públicos justifiquen sus acciones, reaccionen a requerimientos y/o críticas que se les planteen, $\mathrm{y}$ asuman la responsabilidad por sus actos u omisiones, o cuando se apartan de la normativa o de los compromisos asumidos.

Innovación y Tecnología: La idea de que los gobiernos han aceptado y hoy se adhieren a constatar la importancia de proveer 
a la ciudadanía un acceso cada vez más abierto a las nuevas tecnologías, y el relevante rol que ellas tienen en la innovación, así como la importancia de éstas para aumentar la capacidad de los ciudadanos en el uso que de ellas hacen.

\subsection{Elemento de transparencia gubernamental}

Consiste en que la información sobre las actividades de los organismos públicos sea creada y esté a disposición del público, con excepciones limitadas, de manera oportuna y en formatos de datos abiertos sin límites para la reutilización. Esto incluye la divulgación de información en respuesta a las solicitudes de la ciudadanía y de manera proactiva, a iniciativa propia de las entidades públicas. Además de que la información clave acerca de los entes privados esté disponible ya sea directamente o a través de organismos públicos.

Hablar de transparencia no sólo se refiere a establecer una barrera contra la corrupción y los abusos de poder, cosa que sin duda consiguen en buena medida los proyectos de apertura de datos, sino básicamente de un derecho de todo ciudadano a recibir información sobre lo que hacen los gobiernos con el dinero de sus impuestos.

La idea es simple: sólo un ciudadano bien informado de los asuntos públicos puede comprometerse con el desarrollo político y social de su país, sólo un Gobierno radicalmente transparente puede ofrecer a los ciudadanos suficientes datos de su gestión como para que estos puedan opinar y participar con conocimiento de causa y criterio.

Según la open government standars son:

1. Derecho a Saber: Que los gobiernos reconozcan el derecho fundamental de los ciudadanos a acceder a la información, con excepciones limitadas, y que faciliten información en respuesta a las solicitudes y de manera proactiva.

2. Toda la información, de todos los organismos públicos: Que el derecho de acceso a la información sea aplicado a toda la información en poder de organismos nacionales $\mathrm{y}$ supranacionales, incluidos todos los órganos que desempeñan funciones públicas y que operan con fondos públicos (esto incluye a los poderes legislativo y judicial, y las empresas privatizadas que ejercen funciones públicas así como las entidades privadas que posean información relacionada con o que sea necesaria para la protección de los derechos humanos).

3. El acceso es la regla - El secreto es la excepción: La información puede ser retenida sólo si su divulgación pudiera causar un daño demostrable a intereses legítimos de lo permitido por el derecho internacional, y sólo después de considerar el interés público en la dicha divulgación. Estos intereses deben ser protegidos de manera clara y específicamente definidos en la legislación nacional, y debe aplicarse sobre una base de caso por caso. Las mismas excepciones para mantener la información divulgada en respuesta a las solicitudes de acceso a la información y que se describe de forma proactiva, incluso bajo las políticas de datos abiertos.

4. Publicación proactiva: Que las entidades públicas deban publicar de manera proactiva información de interés público, haciendo los esfuerzos necesarios para asegurar el acceso fácil, rápido, efectivo y práctico a dicha información.

5. De carácter gratuito y libre para su reutilización: Que la información se haga pública sin cargo (el estándar internacional prevaleciente es que las solicitudes de información son gratuitas y los únicos cargos que se pueden aplicar son aquellos costes por copiar o costes asociados con la entrega de información) y sin límites en reutilización, incluyendo los impuestos por las licencias $\mathrm{u}$ otras 
restricciones; el derecho a la reutilización de la información pública está en consonancia con el acceso a la información y es parte del derecho fundamental a la libertad de expresión.

6. Formatos Abiertos: La información almacenada electrónicamente debe ser entregada a quienes lo soliciten por vía electrónica y en un formato abierto. La información publicada proactivamente siempre debe estar disponible en formatos abiertos a todo evento.

7. Recopilación de información: que los organismos públicos y las entidades privadas que entran en el ámbito de aplicación del derecho de acceso a la información deban recopilar la información necesaria para la participación pública y la rendición de cuentas. Deben asegurarse de que esta información se compila en una manera oportuna, actualizada periódicamente, y que es clara, completa y comprensible.

8. Mecanismo/Entidad de Supervisión Independiente: Que el derecho de acceso a la información sea supervisado por un organismo independiente que revise su cumplimiento, y que pueda llevar a cabo investigaciones de oficio, recibir y decidir sobre denuncias de los ciudadanos, que esté facultado para ordenar medidas apropiadas asegurando el cumplimiento y la imposición de las sanciones correspondientes si correspondiesen.

\subsection{Elemento de participación ciudadana}

La participación ciudadana es lo que sustenta y sostiene al Gobierno Abierto. Constituye la base de muchos de los otros temas vinculados a las políticas de gobierno abierto, la ciudadanía activa suele ser un enlace vital entre la transparencia y la rendición de cuentas.

Sherry Arnstein (1969) defiende que la participación ciudadana, cuando no es pura impostación, se convierte en un elemento de redistribución del poder, es decir, en un eje transversal a los tres poderes definidos por Montesquieu, quien sitúa al ciudadano como comienzo y final del proceso político, es decir, en su comienzo mediante el voto, eligiendo a sus representantes, $\mathrm{y}$ al final del mismo ejerciendo tareas de control de la gestión e impidiendo desviaciones del mandato. Por lo tanto, aumentar la participación implica redistribuir el poder.

Es importante que la participación ciudadana se diseñe correctamente, cuente con recursos adecuados y nazca del deseo genuino de involucrar al público y de tener en cuenta sus aportaciones. Unos cauces bien diseñados de participación ciudadana pueden promover un funcionamiento democrático más eficiente, legitimar al gobierno, implementar con éxito nuevas medidas y lograr nuevos resultados sociales.

Según la Open Government Standards, los estándares de Participación ciudadana son los siguientes:

1. Apertura: Promover consultas públicas ampliamente, incluso por Internet, listas de correo, anuncios públicos y medios de comunicación, animando a todos a participar y en particular, a los grupos de interés comprometidos.

2. Plazos claros y razonables: Que los procesos participativos estén estructurados de manera de asegurar el tiempo suficiente para permitir que las partes interesadas puedan aprender acerca de la consulta, revisar los materiales, preparar y garantizar la calidad de los aportes considerados.

3. Información completa, clara y comprensible: Que los materiales de apoyo disponibles para los servidores públicos involucrados en los procesos de toma de decisiones se pongan a disposición de los ciudadanos; que los principales datos y su análisis deban ser presentados en una forma que sea accesible y comprensible para el público. 
4. Colaboración activa: Que los organismos públicos sean proactivos en sus interacciones con el público, estableciendo múltiples canales para obtener información (por ejemplo, consultas en línea, audiencias públicas, grupos focales), garantizando así que todos los interesados tengan la oportunidad de participar, y que el debate en torno a un problema pueda evolucionar y madurar con el tiempo.

5. Procedimientos de trabajo claros: Que las reglas sobre la forma de participar en las consultas sean claras, junto con la definición de plazos y cómo deben ser suministrados los comentarios que deberán enviarse a la autoridad pública, así como los lugares y fechas de las audiencias públicas y la manera de obtener invitaciones para asistir y participar.

6. Empoderamiento: Que los procesos participativos tengan por objeto colocar la decisión final en manos del público. Todos los comentarios recibidos deben ser cuidadosamente revisados y las perspectivas presentadas deben incorporarse en la documentación sobre la decisión final. Una justificación detallada debe ser entregada sobre el por qué y el cómo se han tenido en cuenta las opiniones del público.

\subsection{Elemento de rendición de cuentas}

La rendición de cuentas consiste en que existan normas, reglamentos y mecanismos que orienten el comportamiento de las autoridades electas y funcionarios en el ejercicio del poder público y el gasto de los recursos fiscales.

Estas normas deben incluir los requisitos sobre que las decisiones sean plenamente motivadas y justificadas con toda la información puesta a disposición del público. Que exista protección para los denunciantes y mecanismos que permitan reaccionar a las revelaciones de fechorías e irregularidades.

\subsection{Elemento de innovación y tecnología}

La idea de los datos abiertos se sustenta en que los datos deben estar disponibles de manera libre para todos en términos de poder acceder, utilizar y volver a publicar dichos datos, sin restricciones de copyright, patentes $\mathrm{u}$ otros mecanismos de control o propiedad.

La información del sector público puesta a disposición como datos abiertos se denomina "Open Government Data". Los gobiernos y sus contratistas recogen una gran cantidad de datos de alta calidad como parte de sus actividades normales de trabajo. Normalmente, esto se traduce en que el Estado se convierte en un poderoso monopolio de datos capaz de estructurar y homogeneizar las interacciones entre sí y sus ciudadanos.

Estas interacciones unilaterales son caras y no necesariamente responden a las necesidades de los ciudadanos, limitando innecesariamente las actividades del gobierno. La apertura de datos del gobierno implica tanto consideraciones técnicas como políticas.

Un dato es considerado abierto cuando hay:

Disponibilidad y acceso: el dato tiene que estar disponible integralmente y a un costo razonable de reproducción, preferiblemente descargable en la Internet; también tiene que estar disponible en un formato conveniente y modificable.

Reutilización y redistribución: el dato tiene que ser ofrecido en condiciones que permitan la reutilización y redistribución, incluyendo el cruzamiento con otros conjuntos de datos.

Participación universal: todos deben poder usar, reutilizar y redistribuir la información, sin discriminación con las áreas de actuación, personas o grupos. Restricciones "no comerciales" que prevendrían el uso comercial de los datos, o restricciones de uso para ciertos propósitos (por ejemplo "sólo para educación") no son permitidas.

\section{MÉTODO}

Para recoger las impresiones de un primer diagnóstico de gobierno en México se usa el método cualitativo que revisa los resultados 
de la métrica de gobierno abierto 2019 realizada por el centro de investigaciones y docencia económica CIDE y se recogen datos del instituto nacional de estadística y geografía INEGI en contraste con las perspectiva gubernamental y ciudadana.

\section{RESULTADOS}

La transparencia gubernamental, la participación ciudadana, la rendición de cuentas y la tecnología e innovación son los elementos fundamentales para garantizar el gobierno abierto

\section{Figura 1}

Elementos del gobierno abierto

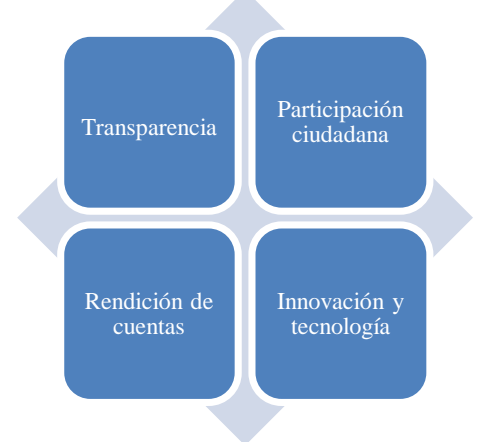

Fuente: elaboración propia a partir de los principios de la alianza para el gobierno abierto

Figura 2

Dimensiones y perspectivas de gobierno abierto

\begin{tabular}{|l|l|l|}
\hline $\begin{array}{l}\text { Perspectiva } \\
\text { gubernamental }\end{array}$ & $\begin{array}{l}\text { Transparencia } \\
\text { ¿El gobierno } \\
\text { hace pública } \\
\text { la } \\
\text { información } \\
\text { relativa a sus } \\
\text { decisiones y } \\
\text { acciones? ¿En } \\
\text { qué medida lo } \\
\text { hace? }\end{array}$ & $\begin{array}{l}\text { qué formas } \\
\text { pueden } \\
\text { incidir las } \\
\text { personas en } \\
\text { las } \\
\text { decisiones } \\
\text { públicas? }\end{array}$ \\
\hline $\begin{array}{l}\text { Perspectiva } \\
\text { ciudadana }\end{array}$ & $\begin{array}{l}\text { ¿Qué tan } \\
\text { factible es } \\
\text { que una } \\
\text { persona } \\
\text { obtenga } \\
\text { información } \\
\text { oportuna y } \\
\text { relevante para }\end{array}$ & $\begin{array}{l}\text { ¿Qué } \\
\text { posibilidad } \\
\text { tienen las } \\
\text { personas } \\
\text { para activar } \\
\text { un } \\
\text { mecanismo } \\
\text { que les } \\
\text { permita }\end{array}$ \\
\hline
\end{tabular}

\begin{tabular}{|l|l|l|}
\hline & $\begin{array}{l}\text { tomar } \\
\text { decisiones? }\end{array}$ & $\begin{array}{l}\text { incidir en las } \\
\text { decisiones } \\
\text { públicas? }\end{array}$ \\
\hline
\end{tabular}

Fuente: inai \& cide metrica de gobierno abierto 2019

El promedio nacional del índice de gobierno abierto 2019 en México, en una escala de 0 a 1 , es de 0.52

\section{CONCLUSIONES}

El promedio nacional del índice de gobierno abierto 2019 en México, en una escala de 0 a 1 , es de 0.52

De donde se desprende que:

- Hay avance en la apertura gubernamental del estado mexicano

- El funcionamiento de los mecanismos de acceso a la información ha mejorado

- El funcionamiento a los mecanismos de acceso a la información ha mejorado. y, finalmente uno de los propósitos centrales del sistema empieza a mostrar resultados: las brechas entre las entidades federativas y entre los tipos de sujetos obligados empiezan a cerrarse.

Sin embargo,

- la ciudadanía aun no encuentra en los sujetos obligados mecanismos formales para participar, que tengan un grado de institucionalización equivalente a aquellos en materia de transparencia: no hay procedimientos claros para las personas que quieren participar, ni capacidad de respuesta efectiva en las oficinas públicas a las iniciativas ciudadanas.

- En materia de participación ciudadana los rezagos son mayores: las formas de incidir en el gobierno no son eficaces para activar los procesos de decisión que permitan incorporar las ideas, demandas o propuestas de la ciudadanía al quehacer gubernamental. 
- Las plataformas para las que la ciudadanía acceda a la información pública funcionan y son utilizadas, pero hay amplias oportunidades para mejorar su diseño y utilidad.

Resultando necesario:

- Fortalecer la lógica de transparencia proactiva, promover el uso de datos abiertos y mejorar la calidad de los portales y los buscadores.

- Lograr la consolidación del sistema nacional de transparencia, acceso a la información pública y protección de datos personales y los avances que llegara a haber en los próximos años en materia de participación ciudadana tengan una lógica que busque servir a las personas para facilitar el ejercicio de sus derechos.

- Para contar con instituciones públicas de calidad se requiere de todos los sectores de la sociedad: ciudadanos, sociedad civil, academia y sector privado.

- La calidad de las instituciones entendidas como la capacidad para resolver o atender los problemas de la población, impacta directamente en los servicios públicos y los programas que atienden. Es decir, influyen en la calidad de vida de las personas.

- La mejora regulatoria es un proceso continuo de construcción de instituciones de calidad que permite atender los problemas de la población, el beneficio a la ciudadanía es directo e inmediato y podría facilitar el acceso a servicios de la población más pobre.

Propuestas imco:

IMCO propone atribuir a la secretaria de la función pública la tarea de evaluar la calidad de las instituciones federales con base a la metodología abierta y robusta, mientras que, en los órdenes estatal y municipal, se puede delegar esta labor a las contralorías locales. Incorporarla mejora regulatoria de tramites y la iniciativa de gobierno abierto en México. Introducir indicadores objetivos y concretar su aplicación permite identificar cuáles son los principales problemas de las instituciones mexicanas y comparar su desempeño con el de otros países.

Retomar las mejores prácticas, diseño institucional, modelo de gobernanza y demás entramado institucional de dependencias como Banxico o el INEGI, las cuales destacan por su calidad. Cabe destacar que ambos organismos cuentan con autonomía técnica y de gestión.

Publicar, para cada organismo, su plan anual de calidad para plantear los retos y definir las funciones de cada dependencia para mejorar su organización y satisfacer las necesidades explicitas e implícitas de los ciudadanos que reciben sus servicios.

Dar cumplimiento y seguimiento a los compromisos de gobierno abierto

Implementar los principios de gobierno abierto de forma transversal en las instituciones públicas, particularmente las que tienen contacto directo con la población.

Retos: Continuar con la operación de registros e indicadores que sean utilizados y nutridos de información para la actual administración pública, contar con registros se traduce en una respuesta a la necesidad de articular, focalizar y monitorear mejora las políticas redistributivas nacionales.

Formar instituciones de calidad que proporcionen productos, servicios, tramites, ayudas de forma exitosa, y sostenida en el tiempo, requiere de comunicación efectiva con los gobernados.

Calidad en las instituciones

¿cómo se mide la calidad en las instituciones? La respuesta abarca un amplio catálogo de características que tienen los organismos públicos, como son: responsabilidades bien definidas, evaluación y mejoramiento de desempeño, procesos transparentes y trabajo en equipo, pero, sobre todo, buenos resultados de la gestión gubernamental y eficiencia para servir a la sociedad. 


\section{REFERENCIAS}

World Bank Group, Doing Business 2019: Training for Reform (Washington, DC.: The World Bank Group), https://espanol.doingbusiness.org/content/dam/doingBusiness/media/AnnualReports/English/DB2019-report_web-version.pdf (Consultado el 07/10/2019). https://imco.org.mx/indices/el-estado-los-estados-y-la-gente/capitulos/resultados/gobierno

Biblioteca de la CEPAl Consultado el 29 de junio de 2020 https://biblioguias.cepal.org/EstadoAbierto/AGA

https://micrositios.inai.org.mx/gobiernoabierto/

Fuente: Naser, Alejandra y Gastón Concha (2012). El desafío hacia el gobierno abierto en la hora de la igualdad; Santiago, CEPAL. p. 30. Documentos de Proyectos No.465 (LC/W.465)

Fuente:Naser, Alejandra y Alvaro Ramírez Alujas, (2013), "Plan de gobierno abierto: Una hoja de ruta para los gobiernos de la región", Santiago, CEPAL. 68

p. Serie ManualesNo. 81, (LC/L.3802 - LC/IP/L.333)

Fuente:Naser, Alejandra y Alvaro Ramírez Alujas, (2013), "Plan de gobierno abierto: Una hoja de ruta para los gobiernos de la región", Santiago, CEPAL. 68

p. Serie ManualesNo. 81, (LC/L.3802 - LC/IP/L.333)

Fuente: Naser, Alejandra y Gastón Concha (2012). El desafío hacia el gobierno abierto en la hora de la igualdad; Santiago, CEPAL. Documentos de Proyectos No.465 (LC/W.465)

Fuente: Naser, Alejandra y Alvaro Ramírez Alujas, (2013), "Plan de gobierno abierto: Una hoja de ruta para los gobiernos de la región", Santiago, CEPAL. 68 p. Serie

Manuales No. 81, (LC/L.3802 - LC/IP/L.333)

Ley general de transparencia y acceso a la información pública, última reforma publicada 13 de agosto de 2020, disponible en:

http://www.diputados.gob.mx/LeyesBiblio/pdf/LGTAIP 130820.pdf

Cejudo, Guillermo M., diccionario de transparencia y acceso a la información, Instituto nacional de transparencia, acceso a la información, y protección de datos personales , mayo de 2019, ISBN: 978-607-98312-3-3 disponible en: https://iacipgto.org.mx/media/pdf/DICCIONARIO\%20DE\%20TRANSPARENCIA\%20INAI.pdf

Alderete, J. L. (2016). "Gobierno Abierto: una visión de sus antecedentes, surgimiento y conceptualización", en Encrucijada Revista Electrónica del Centro de Estudios en Administración Pública. Número 24. Disponible en: http://www. revistas.unam.mx/index.php/encrucijada/article/view/57767/51201

Alianza para el Gobierno Abierto en México. (2020). Lineamientos del Comité Coordinador de la Alianza para el Gobierno Abierto en México. Disponible en: https://drive.google.com/file/d/ 1GGGN3JnGZd8hfm_0MvbRXNaeOndTzD0Y/ view

Calderón, C. y Lorenzo, S. (Coord.). (2010). Open Government: Gobierno Abierto. Algón Editores. Disponible en: http:// libros.metabiblioteca.org:8080/bitstre am/001/163/8/978-84-937218-55.pdf

Centro Latinoamericano de Administración para el Desarrollo (2016). Carta Iberoamericana de Gobierno Abierto. Disponible en: https://clad. org/wp-content/uploads/2020/07/Cartalberoamericana-de-Gobierno-Abierto-07-2016.pdf

Grupo de organizaciones que impulsan la Alianza para el Parlamento Abierto. (2015) Diagnóstico de Parlamento Abierto en México. Disponible en: https://www.parlamentoabierto. mx/wpcontent/themes/parlamentoabierto/ data/Diagn\%C3\%B3stico\%20de\%20Parlamento\%20Abierto.pdf

Insulza, J. M., Zamora, K. C. \& Trigo, M. F. (2014). Gobierno Abierto Municipal en América Latina. De la Proximidad Administrativa a la Acción Colaborativa. Disponible en: http://www.oas.org/ es/sap/dgpe/pub/OEA-Gobierno_Municipal_Abierto.pdf

Open Government Partnership (2020) Manual de OGP. Reglas y Orientación para Participantes. Disponible en: https://www.opengovpartnership.org/wp-content/uploads/2020/02/OGP_ Handbook-Rules-and-Guidance_ES_20200313.pdf 
OCDE/CEPAL/CAF (2016) Perspectivas económicas de América Latina 2017: Juventud, competencias y emprendimiento, OECD Publishing, Paris. Disponible en: https://www.oecd.org/ dev/americas/E-book_LEO2017_SP.pdf

OCDE et al. (2019) Perspectivas económicas de América Latina 2019: Desarrollo en transición, OECD Publishing, Paris, Disponible en: https://doi.org/10.1787/g2g9ff1a-es

Oszlak, O. (2013). Gobierno abierto: hacia un nuevo paradigma de gestión pública. Disponible en: https://www.oas.org/es/sap/ dgpe/pub/coleccion5RG.pdf

Severino, T. \& Moro, J. (2019) Guía de implementación de Gobierno Abierto. Un aporte desde Sociedad Civil. Ciudad de México. Disponible en: https://gobiernoabiertomx.org/ descarga/Guia_implementacion_Gobierno_Abierto_ultima_v.pdf

Instituto nacional de acceso a la información \& centro de investigación y docencia económica: Métrica de gobierno abierto 2019

Instituto mexicano para la competitividad Índice de competitividad internacional 2019

Instituto mexicano para la competitividad Índice de competitividad estatal 2020: estados prevenidos valen por dos. 